\title{
Quality of life in patients with brain metastases using the EORTC QLQ-BN20 and QLQ-C30
}

\author{
Emily Chen • Janet Nguyen • Liying Zhang • \\ Liang Zeng • Lori Holden • Natalie Lauzon • \\ Gillian Bedard • Kaitlin Koo • Alex Mingay • \\ Cyril Danjoux • Arjun Sahgal • May Tsao • \\ Elizabeth Barnes $\cdot$ Edward Chow
}

Received: 31 January 2012 / Accepted: 16 February 2012 /Published online: 2 March 2012

(C) Springer-Verlag 2012

\begin{abstract}
Introduction Given the poor life expectancy of the majority of patients with brain metastases, quality of life (QOL) endpoints are especially valuable to assess in this population. The present study assessed QOL in patients with brain metastases before and after treatment for their disease.

Methods The European Organization for Research and Treatment of Cancer Quality of Life Questionnaire (EORTC QLQ-C30) and the EORTC Brain Cancer Module (EORTC QLQ-BN20) questionnaire were administered to assess the QOL of patients with brain metastases before and 1 month after treatment. Linear regression analysis was applied to assess changes in QOL scores over time and to examine associations between the QLQ-BN20 and QLQ-C30 scales, patient demographics and clinical variables. Associations between the QLQ-BN20 and QLQ-C30 scales were evaluated using Spearman correlation.

Results There were 47 patients assessed at baseline, 31 (67\%) completed follow-up at 1 month post-treatment. The majority $(81 \%)$ of patients received whole-brain radiotherapy only. Future uncertainty (QLQ-BN20) and fatigue (QLQC30) were the most prominent symptoms at baseline. Most
\end{abstract}

E. Chen $\cdot$ J. Nguyen $\cdot$ L. Zhang $\cdot$ L. Zeng $\cdot$ L. Holden $\cdot$

N. Lauzon $\cdot$ G. Bedard $\cdot$ K. Koo $\cdot$ A. Mingay $\cdot$ C. Danjoux $\cdot$

A. Sahgal $\cdot$ M. Tsao $\cdot$ E. Barnes $\cdot$ E. Chow

Rapid Response Radiotherapy Program, Odette Cancer Centre,

Sunnybrook Health Sciences Centre, University of Toronto,

Toronto, ON, Canada

E. Chow $(\square)$

Department of Radiation Oncology, Odette Cancer Centre,

Sunnybrook Health Sciences Centre,

2075 Bayview Avenue,

Toronto, ON, Canada M4N 3M5

e-mail: edward.chow@sunnybrook.ca
QLQ-BN20 and QLQ-C30 scales did not significantly change from baseline to follow-up with the exception of hair loss $(p=0.0004)$ and itchy skin $(p<0.0001)$, which worsened post-treatment. Baseline KPS was positively correlated with QLQ-C30 physical functioning scale but negatively correlated to QLQ-BN20 motor dysfunction $(p=0.016)$, hair loss $(p=0.013)$ and leg weakness $(p=0.015)$ as well as QLQ-C30 pain $(p=0.042)$ and appetite loss $(p=0.030)$.

Conclusion The maintenance of nearly all QOL scores 1 month after treatment indicates the treatment intervention likely played a symptom-stabilizing role and prevented QOL deterioration in the palliative setting.

Keywords Brain metastases · Palliative radiation - Quality of life $\cdot$ QLQ-BN20 · QLQ-C30

\section{Introduction}

Brain metastases constitute the most common intracranial neoplasm in adults, occurring in $10-15 \%$ of patients with advanced cancer $[1,2]$. The majority of brain metastases originate from primary cancer of the lung (40-50\%), breast (15-25\%) or skin $(5-20 \%)[3,4]$. Depending on the location of the brain metastases, patients may present with a range of neurological symptoms such as headaches, focal weakness, mental disturbances, behavioural changes, seizures, speech difficulty, and ataxia [5].

The management of brain metastases depends on several patient-related and tumour-related characteristics [6]. Whole-brain radiotherapy (WBRT) and/or corticosteroids are the standard treatment for the majority of patients presenting with multiple lesions or widespread metastatic disease. More aggressive treatment modalities such as surgery 
and stereotactic radiosurgery (SRS) are reserved for patients with good performance status and limited extracranial disease. With treatment, median survival increases up to 3 to 6 months compared to the median survival of 1 month among untreated patients with brain metastases $[5,7,8]$. While traditional endpoints of survival and local brain tumour control are important to consider, quality of life (QOL) is arguably the more important endpoint to assess given the guarded prognosis of this patient population.

QOL refers to a subjective multi-dimensional construct encompassing physical, emotional, social and cognitive functions. The European Organization for Research and Treatment of Cancer core questionnaire (EORTC QLQ-C30) is an internationally validated instrument frequently used to assess QOL in oncology clinical trials [9]. The EORTC Quality of Life Questionnaire-Brain Neoplasm (QLQ-BN20) is a 20-item questionnaire developed and validated for patients with primary brain tumours $[10,11]$. This cancer subtype-specific QOL questionnaire is often used as a supplement to the QLQ-C30 core general questionnaire. The present study assessed QOL in patients with brain metastases before and after treatment using the QLQ-BN20 in conjunction with the QLQ-C30.

\section{Methods and materials}

\section{Patients}

Patients undergoing WBRT, radiosurgery/gamma knife and/ or neurosurgical resection, for their brain metastases were approached for the study. English-speaking patients over the age of 18 with documented single or multiple brain metastases were eligible for this study. Consenting patients completed the QLQ-BN20 and QLQ-C30 questionnaires before and 1 month after treatment for brain metastases. Baseline demographic data collected included age, Karnosky performance status (KPS), gender, primary cancer site and the presence of visceral or bone metastases. All research encompassed in this study was approved by the Sunnybrook Health Sciences Centre research ethics board.

\section{Questionnaires}

The EORTC QLQ-C30 and QLQ-BN20 questionnaires were completed by patients at baseline and 1 month following the treatment with a research assistant. The QLQ-C30 is a 30-item general questionnaire that assesses a wide range of functional outcomes and symptoms relevant among oncology patients. It consists of five functional domains assessing physical role, cognitive, emotional, and social aspects, one global QOL domain, three symptom domains (fatigue, pain, and nausea and vomiting), five single items assessing other symptoms (dyspnea, insomnia, anorexia, constipation, and diarrhea), and one item assessing financial impact. Each question/item was scored on a numeric scale from 1 to $4(1=$ "not at all"; 2 = "a little"; 3 = "quite a bit"; 4 = "very much"). The only exception was with last two items assessing overall health and overall QOL, both of which were scored from 1 (very poor) to 7 (excellent). Scores $(0-100)$ for each scale and the single items were generated according to the QLQ-C30 scoring manual [12]. On the scale of $0-100$, a higher score for each symptom scale corresponds to worse symptom burden. Conversely, higher scores are favourable for functional scale items and the last two QLQ-C30 questions assessing patient's perceived overall health and overall QOL.

The QLQ-BN20 consists of 20 questions; seven single item symptom scales (headaches, seizures, drowsiness, hair loss, itchy skin, leg weakness and bladder control), along with four multi-item scales (future uncertainty, visual disorder, motor dysfunction and communication deficit). Raw scores for the QLQ-BN20 items were computed and subsequently transformed linearly to a $0-100$ scale. Procedures for scale and single item scoring were similar to that of the EORTC-C30 questionnaire. A higher score represents worse QOL for all QLQ-BN20 scales and single items.

\section{Statistical analysis}

Descriptive statistics summarized demographics. Spearman correlation was used to explore relationships between QLQBN20/QLQ-C30 scales at baseline and post brain metastases treatment. Mean QLQ-BN20 and QLQ-C30 were plotted for baseline and follow-up. One-way analysis of variance (ANOVA) was applied to compare QLQ-C30 and QLQ BN20 scores between baseline and follow-up visits. To normalize the distribution of each QLQ score, data were transformed using natural $\log$. In the ANOVA, the outcome was the log-transformed QLQ score and the independent variable was the binary visit variable (baseline or follow-up). Additionally, general linear regression analysis was used to examine the association between KPS and QLQ-BN20/QLQ-C30 scores at baseline and follow-up, respectively. A two-sided $p$ value $<0.05$ was considered statistically significant. All analyses were conducted using Statistical Analysis Software (SAS for Windows, version 9.2).

\section{Results}

A total of 47 patients completed the QLQ-BN20 and QLQC30 questionnaires at initial consultation. Baseline patient characteristics of the study population are summarized in Table 1 . The most common primary cancers were of the lung $(37 \%)$ and breast $(35 \%)$. The majority of patients received WBRT alone (81\%), while the remaining patients received either WBRT with radiosurgery $(15 \%)$ or neurosurgery $(4 \%)$. 
Table 1 Baseline patient characteristics $(n=47)$

\begin{tabular}{|c|c|}
\hline \multicolumn{2}{|l|}{ Age (years) } \\
\hline$n$ & 47 \\
\hline Mean \pm SD & $60.6 \pm 11.9$ \\
\hline Inter-quartiles & $52-69$ \\
\hline Median (range) & $61(24-86)$ \\
\hline \multicolumn{2}{|l|}{ KPS } \\
\hline$n$ & 45 \\
\hline Mean \pm SD & $70.4 \pm 13.3$ \\
\hline Inter-quartiles & $70-80$ \\
\hline Median (range) & $70(40-100)$ \\
\hline \multicolumn{2}{|l|}{ Gender } \\
\hline Female & $27(57 \%)$ \\
\hline Male & $20(43 \%)$ \\
\hline \multicolumn{2}{|l|}{ Married status } \\
\hline Married & $35(74 \%)$ \\
\hline Widowed & $6(13 \%)$ \\
\hline Single & $5(11 \%)$ \\
\hline Other & $1(2 \%)$ \\
\hline \multicolumn{2}{|l|}{ Primary cancer site } \\
\hline Lung & $17(37 \%)$ \\
\hline Breast & $16(35 \%)$ \\
\hline Colon & $3(7 \%)$ \\
\hline Liver & $2(4 \%)$ \\
\hline Renal cell/kidney & $2(4 \%)$ \\
\hline Stomach & $2(4 \%)$ \\
\hline Endometrial & $1(2 \%)$ \\
\hline Oesophagus & $1(2 \%)$ \\
\hline Ovarian & $1(2 \%)$ \\
\hline Unknown & $1(2 \%)$ \\
\hline \multicolumn{2}{|l|}{ Number of brain metastases } \\
\hline 1 & $14(33 \%)$ \\
\hline 2 to 3 & $15(36 \%)$ \\
\hline$>3$ & $13(31 \%)$ \\
\hline \multicolumn{2}{|l|}{ Treatment } \\
\hline Neurosurgery alone & $2(4 \%)$ \\
\hline Radiosurgery/gamma knife \pm WBRT $^{\mathrm{a}}$ & $7(15 \%)$ \\
\hline WBRT alone & $38(81 \%)$ \\
\hline \multicolumn{2}{|l|}{ Previous systemic therapy } \\
\hline Yes & $31(66 \%)$ \\
\hline No & $16(34 \%)$ \\
\hline
\end{tabular}

${ }^{a}$ Patient received whole-brain radiotherapy (WBRT) and radiosurgery within 4 weeks of one another, and both treatments were prior to the 1 month follow-up

Of the initial 47 patients, $30(67 \%)$ completed both questionnaires at follow-up.

All QLQ-BN20 baseline scales other than "difficulty concentrating" significantly correlated with at least one QLQ-C30 scale at baseline (Table 2). Conversely, all QLQ-C30 scales significantly correlated with one or more
QLQ-BN20 scale at baseline. Overall QOL was significantly negatively correlated with QLQ-BN20 scales of future uncertainty, motor dysfunction and headaches.

When comparing baseline and follow-up questionnaire scales, significant worsening from baseline was only observed with QLQ-BN20 hair loss $(p=0.0004$; 8.3 vs. 33.3 for baseline or follow-up mean scale) and itchy skin $(p<0.0001 ; 0$ vs. 21.8 for baseline or followup mean scale) scores. The global QOL scale as assessed by the QLQ-C30 was not significantly different at follow-up compared to baseline, indicating the treatment intervention served to maintain QOL in the study population. No other significant improvement or deterioration was observed in the remaining QLQ-BN20 and QLQ-C30 scales.

For this population, none of the patients experienced itchy skin at baseline. Mean baseline symptom scores (Fig. 1) for the remaining QLQ-BN20 scales ranged from $8 \pm 21$ (seizures) to $45 \pm 26$ (future uncertainty). QLQ-C30 mean symptom scores (Fig. 2) at baseline ranged from $4 \pm 16$ (diarrhea) to $44 \pm 28$ (fatigue), with higher scores indicating greater symptom burden. QLQ-C30 mean baseline scores for the functional scales and overall QOL ranged from $55 \pm$ 29 (role functioning) to $73 \pm 24$ (cognitive functioning). Higher scores for these functional scales are reflective of more favourable outcome.

At baseline, initial KPS was negatively correlated to QLQ-BN20 scales of motor dysfunction $(p=0.016)$, hair loss $(p=0.013)$ and leg weakness $(p=0.015)$. In other words, patients with a lower KPS experienced greater symptom burden in these scales. A negative correlation was observed with KPS and QLQ-C30 scales of pain $(p=0.042)$ and appetite loss $(p=0.030)$. However, patients with higher KPS were more likely to have better physical functioning $(p=0.0007)$.

The mean follow-up QLQ-BN20 and QLQ-C30 scores are displayed in Figs. 1 and 2, respectively. Mean QLQBN20 symptom scores at follow-up ranged from $8 \pm 21$ (seizures) to $44.2 \pm 26$ (future uncertainty). QLQ-C30 mean symptom scores at follow-up ranged from $4.9 \pm 15$ (dyspnea) to $49 \pm 25$ (fatigue). Mean QLQ-C30 functional scale and overall QOL scores ranged from $56 \pm 21$ (global health status/QOL) to $71 \pm 26$ (cognitive functioning). Lower symptom scores and higher functioning scores indicate more favourable outcome.

At follow-up visit, initial KPS was negatively related to QLQ-BN20 scales of difficulty concentrating $(p=0.030)$, visual disorder $(p=0.017)$, motor dysfunction $(p=0.046)$, seizures $(p=0.011)$ and bladder control $(p=0.0008)$. This revealed that patients with lower initial KPS were more likely to experience greater symptom burden in these scales. Conversely, better baseline KPS was associated with better physical functioning $(p=0.011)$. 


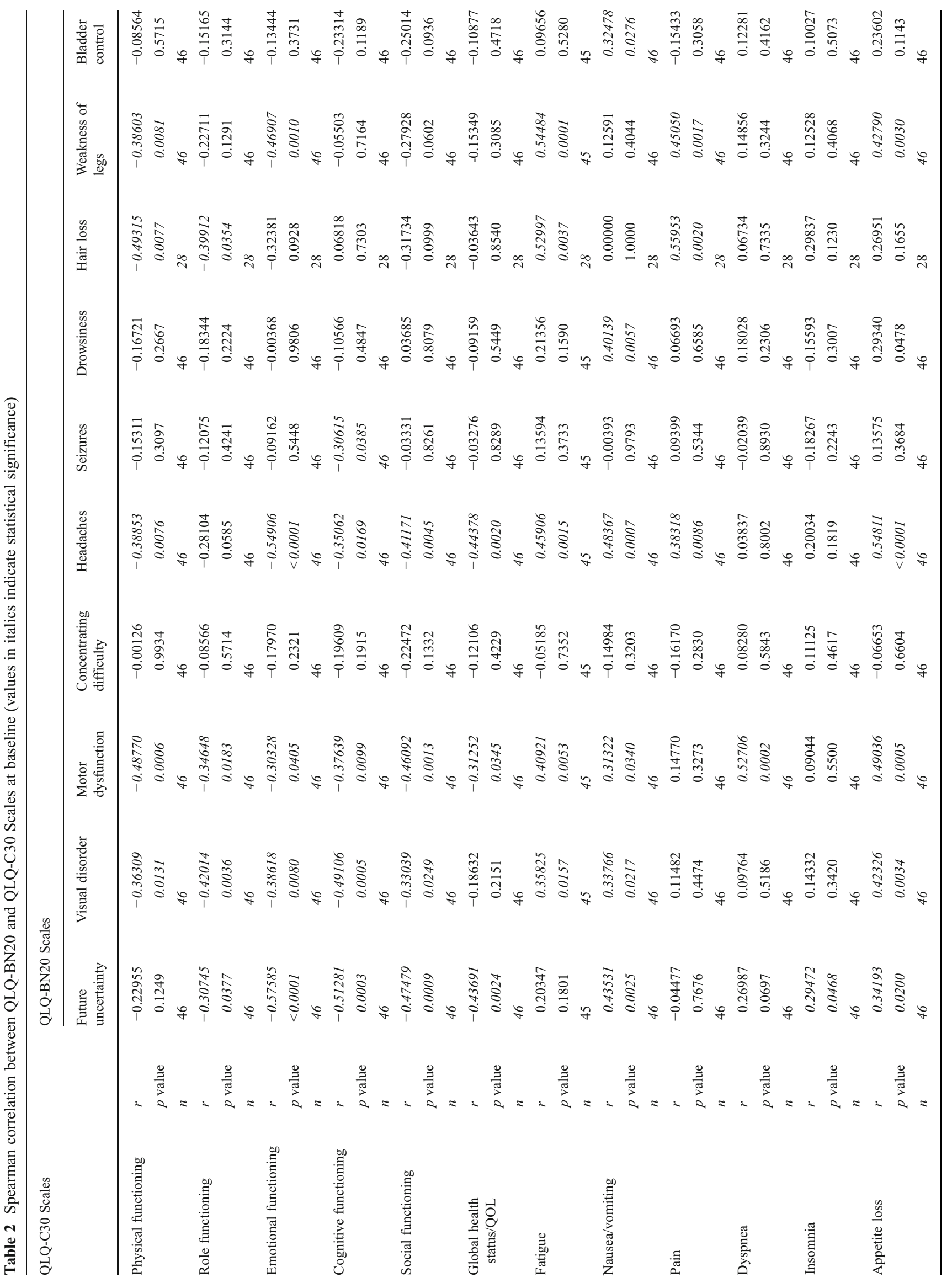




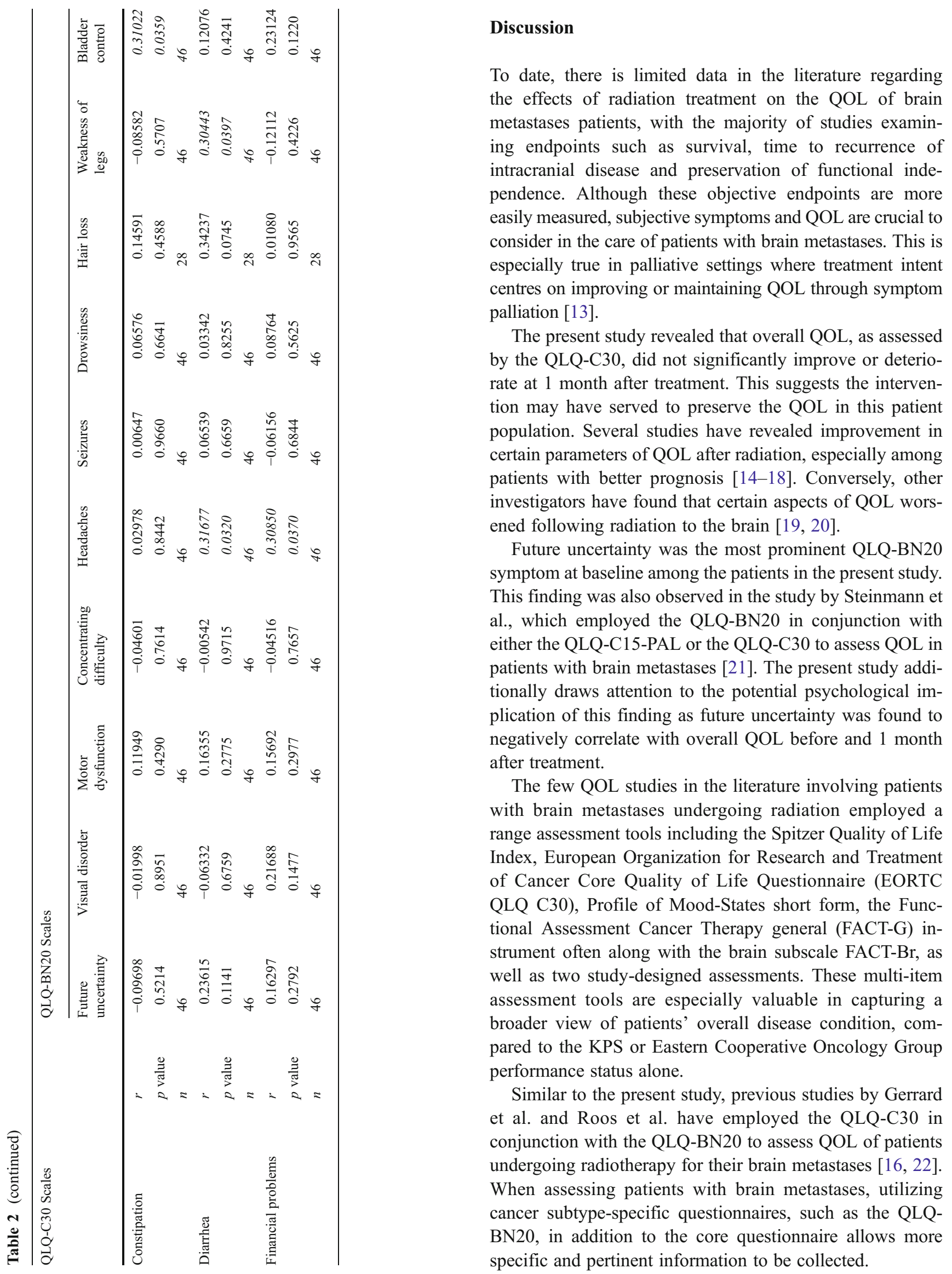


Fig. 1 EORTC QLQ-BN20

mean scores before and after radiotherapy. A higher score indicates worse symptomatology for all scales; bars represent standard errors; stars indicate statistical significance

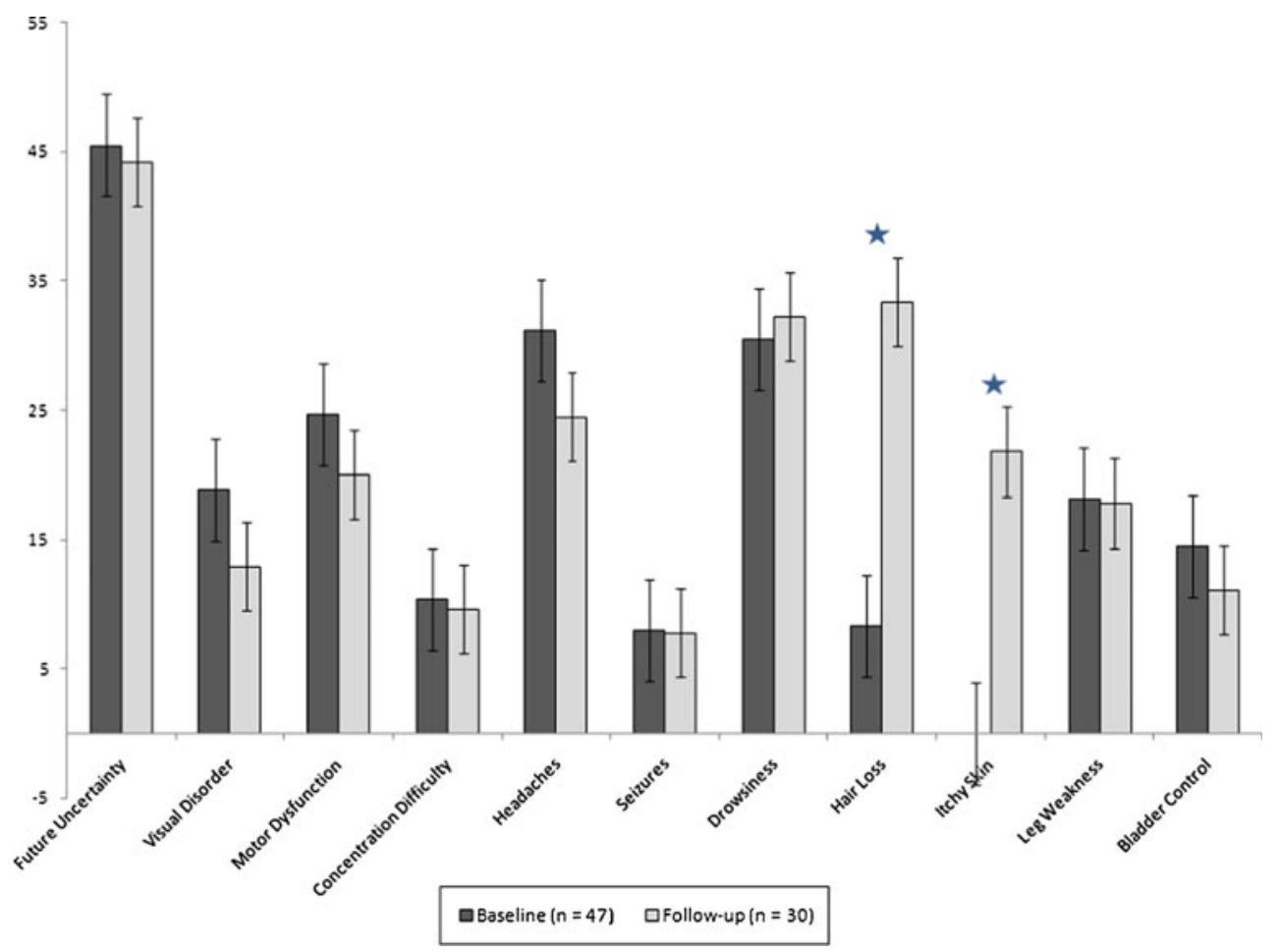

Gerrard et al. performed three studies aimed to evaluate WBRT in patients with brain metastases, two of which employed the QLQ-BN20 and QLQ-C30 questionnaires
[22]. The first study assessed QOL in patients with multiple brain metastases and poor prognostic features receiving WBRT. The two questionnaires were completed before

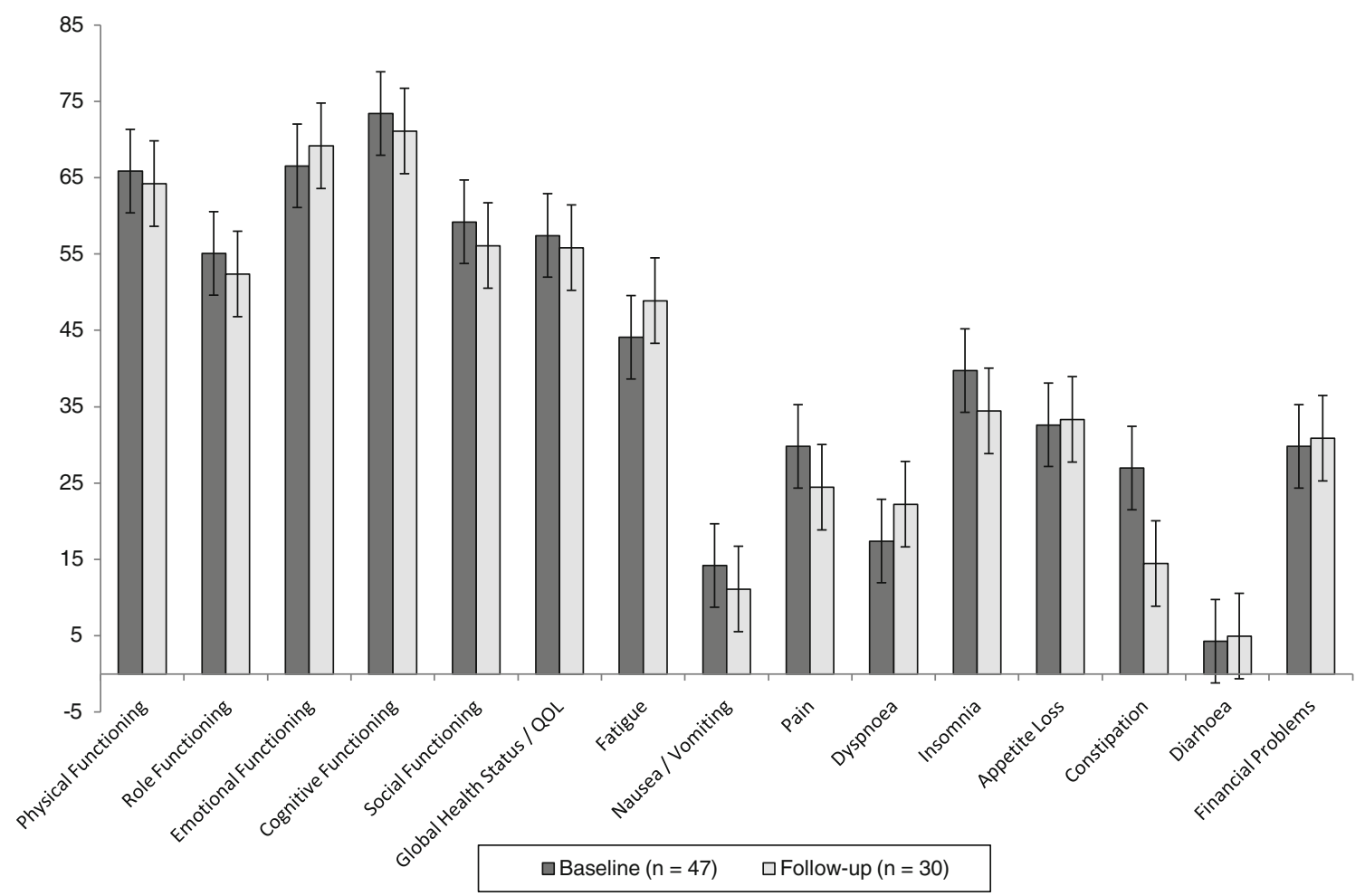

Fig. 2 EORTC QLQ-C30 mean scores before and after radiotherapy. Higher score indicates worse symptomatology for all symptom scales. A higher score indicates better functioning/quality of life for all functioning and quality of life scales; bars indicate standard errors, no items were statistically significant 
WBRT as well as 2, 4 and 8 weeks after treatment. Data analysed from 18 patients revealed high levels of drowsiness and fatigue that persisted from baseline to 8 weeks. Poor levels of physical and social functioning were also noted among patients in this study. Improvement in QOL was only observed in three patients at either 2 or 4 weeks posttreatment. In their second study, Gerrard et al. examined QOL in patients with brain metastases receiving WBRT (20 Gy/5 as opposed to $12 \mathrm{~Gy} / 2$ in the first study). Unfortunately, due to poor recruitment and assessment difficulties, the study was terminated prematurely with only six patients accrued. Data collected at baseline as well as 4 and 8 weeks postWBRT did not show any evidence of QOL improvement in this patient sample.

The randomized study of WBRT vs. observation (control group) after surgery or post radiosurgery by Roos et al. also used the QLQ-BN20 and QLQ-C30 to examine QOL longitudinally [15]. Due to slow accrual, the investigators terminated the study prematurely with a total of 19 patients accrued. Detailed QOL analysis was not conducted as a result of the small sample size. Data from the 19 patients did not reveal significant differences in the two study arms in terms of global health scores or global QOL scores. The results of this study did not indicate any significant improvement or deterioration after patients received WBRT.

The three studies by Gerrard et al. and Roos et al. experienced difficulty with data collection, especially at later followups $[16,22]$. High attrition rates were also noted in other similar QOL studies involving patient with brain metastases using either the FACT-Br alone or in conjunction with the FACT-G [20,23]. As expected due to the guarded prognosis and rapid deterioration of this patient cohort, many patients (23\%) were also lost at follow-up in the present study. Although QOL assessment is anticipated to be difficult in this population, further efforts towards maximizing recruitment and compliance at subsequent follow-ups should be made. While the QLQ-BN20 and QLQ-C30 used in this study provide a comprehensive overview of QOL, the task of completing 50 questionnaire items can be quite arduous for patients with poor performance status and mental status. In the multicentre pilot study by Steinmann et al., examining the effects of radiotherapy for brain metastases on quality of life, patients were either asked to complete the QLQ-C30 or QLQ-C15PAL in addition to the QLQ-BN20 [21]. The EORTC QLQC15-PAL is a 15-item version of the 30-item QLQ-C30, designed especially for palliative cancer patients with the intent of decreasing assessment burden among the advanced cancer patient population. In comparing the use of the two questionnaire combinations, notably higher compliance and practicability resulted with the shorter version (QLQ-C15PAL). Future QOL studies involving patients with brain metastases may likewise benefit from the utilization of more condensed, yet still comprehensive questionnaires.
A limitation in the present study was the attrition due to health deterioration or death, as observed in similar studies [24]. As such, the findings of the study may be more representative of patients with better prognosis who were well enough to complete follow-up. Also, systemic treatments post-radiotherapy were not recorded which may have confounded our findings. Brain metastases constitute a serious and debilitating complication in many cancer patients. In evaluating the effectiveness of treatment options among this patient population with limited life expectancy, assessing QOL may uncover more informative findings compared to measuring traditional end points such as survival alone. It is important to note that the QOL issues experienced by patients with brain metastases may not completely mirror those experienced by patients with primary brain neoplasm. Therefore, further studies validating QOL questionnaires initially developed for primary brain cancer patients, such as the QLQBN20 and the FACT-Br, among patients with brain metastases are necessary. Understanding the expected QOL outcomes of WBRT and other available treatment modalities for this patient population may prove instrumental in making optimal treatment decisions.

Acknowledgement We thank the generous support of Bratty Family Fund, Michael and Karyn Goldstein Cancer Research Fund, Joseph and Silvana Melara Cancer Research Fund, and Ofelia Cancer Research Fund.

Conflict of interest The authors have no conflict of interest to disclose.

\section{References}

1. Chamberlain MC (2010) Brain metastases: a medical neurooncology perspective. Expert Rev Neurother 10:563-573

2. Sperduto PW et al (2010) Diagnosis-specific prognostic factors, indexes, and treatment outcomes for patients with newly diagnosed brain metastases: a multi-institutional analysis of 4,259 patients. Int J Radiat Oncol Biol Phys 77:655-661

3. Schouten LJ, Rutten J, Huveneers HA et al (2002) Incidence of brain metastases in a cohort of patients with carcinoma of the breast, colon, kidney, and lung and melanoma. Cancer 94:2698-2705

4. Barnholtz-Sloan JS, Sloan AE, Davis FG et al (2004) Incidence proportions of brain metastases in patients diagnosed (1973 to 2001) in the Metropolitan Detroit Cancer Surveillance System. J Clin Oncol 22:2865-2872

5. Posner JB (1977) Management of central nervous system metastasis. Semin Oncol 4:81-91

6. Lohr F, Pirzkall A, Hof H et al (2001) Adjuvant treatment of brain metastases. Semin Surg Oncol 6:543-551

7. Chao JH, Phillips R, Nickson JJ (1954) Roentgen-ray therapy of cerebral metastases. Cancer 7(4):682-689

8. Zimm S, Wampler GL, Stablein D et al (1981) Intracerebral metastases in solid-tumor patients: natural history and results of treatment. Cancer 48(2):384-394

9. Aaronson NK, Ahmedzai S, Bergman B et al (1993) The European organization for research and treatment of cancer QLQ-C30: a quality-of-life instrument for use in international clinical trials in oncology. J Natl Cancer Inst 85:365-376 
10. Osoba D, Aaronson NK, Muller M et al (1996) The development and psychometric validation of a brain cancer quality-of-life questionnaire for use in combination with general cancer-specific questionnaires. Qual Life Res 5:139-150

11. Taphoorn MJ, Claassens L, Aaronson NK et al (2010) An international validation study of the EORTC brain cancer module (EORTC QLQ-BN20) for assessing health-related quality of life and symptoms in brain cancer patients. Eur J Cancer 46:1033-1040

12. Fayers PM, Aaronson NK, Bjordal K, Groenvold M, Curran D, Bottomley A et al (2001) The EORTC QLQ-C30 scoring manual, 3rd edn. European Organisation for Research and Treatment of Cancer, Brussels

13. Kirkbride P, Tannock IF (2008) Trials in palliative treatmenthave the goal posts been moved? Lancet Oncol 9:186-187

14. Wong J, Hird A, Zhang L et al (2009) Symptoms and quality of life in cancer patients with brain metastases following palliative radiotherapy. Int J Radiat Oncol Biol Phys 75(4):1125-1131

15. Roos DE, Wirth A, Burmeister BH et al (2006) Whole brain irradiation following surgery or radiosurgery for solitary brain metastases: Mature results of a prematurely closed randomized Trans-Tasman Radiation Oncology Group trial (TROG 98.05). Radiother Oncol 80(3):318-322

16. Addeo R, Caraglia M, Faiola V, Kirkbride P, Tannock IF (2008) Trials in palliative treatment-have the goal posts been moved? Lancet Oncol 9:186-187

17. Scott C, Suh J, Stea B, Nabid A, Hackman J (2007) Improved survival, quality of life, and quality-adjusted survival in breast cancer patients treated with efaproxiral (Efaproxyn) plus whole brain radiation therapy for brain metastases. Am J Clin Oncol 30:580-587

18. Yaneva MP, Semerdjieva MA (2006) Assessment of the effect of palliative radiotherapy for cancer patients with intracranial metastases using EORTC-QOL-C30 questionnaire. Folia Medica 48:2329

19. Chow E, Davis L, Holden L, Tsao M, Danjoux C (2005) Prospective assessment of patient-rated symptoms following whole brain radiotherapy for brain metastases. J Pain Symptom Manag 30:18-23

20. Bezjak A, Adam J, Barton R et al (2002) Symptom response after palliative radiotherapy for patients with brain metastases. Eur $\mathrm{J}$ Cancer 38:487-496

21. Steinmann D, Schafer C, van Oorschot B et al (2009) Effects of radiotherapy for brain metastases on quality of life $(\mathrm{QoL})$. Prospective pilot study of the DEGRO QoL working party. Strahlenther Onkol 185:190-197

22. Gerrard GE, Prestwich RJ, Edwards A et al (2003) Investigating the palliative efficacy of whole-brain radiotherapy for patients with multiple-brain metastases and poor prognostic features. Clin Oncol $15: 422-428$

23. Doyle M, Bradley NM, Li K et al (2007) Quality of life in patients with brain metastases treated with a palliative course of wholebrain radiotherapy. J Palliat Med 10(2):367-374

24. Wong J, Hird A, Kirou-Mauro A, Napolskikh J, Chow E (2008) Quality of life in brain metastases radiation trials: a literature review. Curr Oncol 15(5):25-45 